\title{
The Diagnostic Value of Alarm Features for Identifying Types and Stages of Upper Gastrointestinal Malignancies
}

\author{
Shou-Wu Lee ${ }^{\text {a, b, d }}$, Chi-Sen Chang ${ }^{\mathrm{a}, \mathrm{b}}$, Hong-Jeh Yeh ${ }^{\mathrm{a}, \mathrm{c}}$, Han-Chung Lien ${ }^{\mathrm{a}, \mathrm{c}}$, \\ Teng-Yu Lee ${ }^{a}$, b, Yen-Chun Peng ${ }^{a, c}$
}

\begin{abstract}
Background: Upper gastrointestinal (GI) malignancies are an uncommon cause of dyspepsia but of great concern. The aim of this study was to determine the association between alarm features and each type and stage of upper GI malignancies.

Methods: Patients who underwent endoscopy for symptoms of dyspepsia between January 2008 and December 2009 were retrospectively collected. Alarm features studied in this study were dysplasia, body weight loss and GI bleeding. Patients were classified according to the findings of endoscopy and histological reports.
\end{abstract}

Results: A total of 3,926 patients were included in the study, with $82(2.1 \%)$ cases with GI malignancies. The specificity and negative predictive value of alarm features ranged from $93.8 \%$ to $99.8 \%$, but the sensitivity and positive predictive value ranged from $11.6 \%$ to $29.3 \%$. The only variable with a positive predictive value was dysphagia $(66.7 \%)$. The patients with esophageal cancers and upper gastric cancers had the highest ratio of alarm features, most body weight loss and dysphagia. There was a positive correlation between alarm features and advanced stages of gastric cancers, with the exception of GI bleeding sign.

Conclusion: Although alarm features had a low sensitivity in identifying patients with upper GI malignancies, the presence of alarm features did help diagnose esophageal or upper gastric cancer and the sign of GI bleeding for early gastric cancer. In addition, dysphagia and weight loss are associated with higher stages of gastric cancer.

Keywords: Alarm features; Gastrointestinal; Malignancy

Manuscript accepted for publication April 03, 2017

aDivision of Gastroenterology, Department of Internal Medicine, Taichung Veterans General Hospital, Taichung, Taiwan, Republic of China

${ }^{b}$ Department of Internal Medicine, Chung Shan Medical University, Taichung, Taiwan, Republic of China

'Department of Internal Medicine, Yang-Ming University, Taipei, Taiwan, Republic of China

${ }^{\mathrm{d} C}$ Corresponding Author: Shou-Wu Lee, Division of Gastroenterology, Department of Internal Medicine, Taichung Veterans General Hospital, Taichung, 1650 Taiwan Boulevard, Sec. 4, Taichung 40705, Taiwan, Republic of China. Email: ericest@vghtc.gov.tw

doi: https://doi.org/10.14740/gr826w

\section{Introduction}

Dyspepsia is a common condition in clinical practice and is frequently encountered by primary care physicians and specialists $[1,2]$. Upper gastrointestinal (GI) malignancies are an uncommon cause of dyspepsia, but the treating physician must nevertheless rule them out [2]. It is vitally important not to delay diagnosis in patients with an underlying malignant disease. Therefore, patients who have a high risk of developing cancer must receive an immediate endoscopic examination to rule out malignancy [3].

Alarm features, also called alert features, red flags, or warning signs, are specific features thought to be associated with serious gastroenterologic disease, which may include underlying malignancy or significant pathology such as a stricture or ulcer [2]. Alarm symptoms are generally accepted as an indication for direct endoscopy. This is reflected in several guidelines for treatment of patients with dyspeptic symptoms. However, previous studies noted that the sensitivity of alarm features for predicting cases with upper GI malignancies is unsatisfactory [2-4]. Furthermore, most previous studies have investigated populations in western countries, where prevalence of upper GI malignancies is low. Furthermore, to the best of our knowledge, the relationships between alarm features and stages of GI cancer have not been investigated. The aim of this study was to determine the associations between alarm features and types and stages of upper GI malignancy in Chinese patients.

\section{Patients and Methods}

Data from medical records of consecutive patients who underwent open-access upper endoscopy for symptoms of dyspepsia in our hospital, an academic urban tertiary-care center, were retrospectively collected between January 2008 and December 2009. Dyspepsia was defined as pain and discomfort centered in the upper abdomen. Exclusion criteria were as follows: 1) cirrhosis with varices or portal hypertensive gastropathy, 2) acid regurgitation or heartburn sensation as the main symptom, 3) prior gastric surgery, and 4) use of anti-acid medication, such as a proton pump inhibitor (PPI) or an H2-receptor antagonist (H2RB), or other medications, including aspirin and other nonsteroidal anti-inflammatory drugs (NSAIDs) or 
Table 1. The Basic Characteristics of Enrolled Individuals

\begin{tabular}{|c|c|c|c|c|c|}
\hline & \multicolumn{5}{|c|}{ Upper GI endoscopy finding } \\
\hline & $\begin{array}{l}\text { Normal } \\
(n=2,642,67.3 \%)\end{array}$ & $\begin{array}{l}\text { Gastroduodenitis/ } \\
\text { peptic ulcer disease } \\
(n=1,202,30.6 \%)\end{array}$ & $\begin{array}{l}\text { Upper GI } \\
\text { malignancies } \\
(\mathrm{n}=\mathbf{8 2}, \mathbf{2 . 1 \%})\end{array}$ & Total $(n=3,926)$ & P-value \\
\hline Age (years) & $47.5 \pm 15.7$ & $54.6 \pm 14.3$ & $64.6 \pm 13.5$ & $50.3 \pm 16.2$ & $0.001^{\mathrm{a}}$ \\
\hline \multicolumn{6}{|l|}{ Gender } \\
\hline Male & $1,134(42.9 \%)$ & $644(53.6 \%)$ & $58(70.7 \%)$ & $1,836(46.8 \%)$ & $0.001^{\mathrm{b}}$ \\
\hline \multicolumn{6}{|l|}{ H. pylori infection } \\
\hline Positive & $296(17.1 \%)$ & $522(43.4 \%)$ & $12(17.1 \%)$ & $421(28.4 \%)$ & $0.001^{\mathrm{b}}$ \\
\hline Excluded numbers & 906 & 90 & 12 & & \\
\hline \multicolumn{6}{|l|}{ Alarm features } \\
\hline At least one alarm feature & $102(3.9 \%)$ & $138(11.5 \%)$ & $50(60.1 \%)$ & $290(7.4 \%)$ & $0.001^{\mathrm{b}}$ \\
\hline
\end{tabular}

aKruskal-Wallis one-way ANOVA test. 'bearson's Chi-square test. Sixteen patients had more than one alarm features.

coumadin, for more than 2 months. Written informed consent for upper endoscopy was obtained from all patients before the procedure.

Alarm symptoms were recorded and included dysphagia, defined as the perception of an impediment to the normal passage of swallowed material, unexplained weight loss of more than $5 \mathrm{~kg}$ in 2 months, and signs of GI bleeding such as melena, positive fecal occult blood test, or hematemesis within the preceding month. Helicobacter pylori status was determined by rapid urease test (CLO test, Delta West, Bentley, Australia) using a sample obtained from antral biopsy, and testing was done at the discretion of primary gastroenterologists. Patients were classified according to the findings of upper endoscopy, which were confirmed by two experienced gastroenterologists, and upper GI malignancy was additionally confirmed by histological study. Staging of gastric cancers, including Bormann's [5], histological [6] and AJCC classification eighth edition [7], was performed based on endoscopic findings, histopathological studies and computed tomography.

Data of age were expressed as the standard deviation of the mean for each of the measured parameters. Gender, H. pylori infection, and alarm features were expressed as percentages of the total number of patients. A P value below 0.05 was considered statistically significant. Statistical comparisons were made using Pearson's Chi-square test or Fisher's exact test to compare gender, H. pylori infection, and alarm features. Mann-Whitney U test was used for analysis of the age distributions among patient groups.

\section{Results}

The data of 4,550 patients who underwent endoscopy between January 2008 and December 2009 were collected. Among these patients, 380 and 244 cases were excluded due to varices and prior gastric surgery, respectively. In total, 3,926 consecutive patients were included in the final analysis, as shown in Table 1. Among these patients, 2,642 patients (67.3\%) had normal endoscopic findings. The diagnosis of upper GI malignancies, which was made by open-access upper endoscopy and confirmed by histopathological study, accounted for 82 cases $(2.1 \%)$. The remaining 1,202 patients $(30.6 \%)$ had endoscopic findings of gastroduodenitis or peptic ulcer disease.

The mean age of patients with upper GI malignancies (64.6 years) was significantly higher among those with gastroduodenitis and peptic ulcer disease (54.6 years) and in those with normal endoscopic findings (47.5 years). There were more male than female patients with upper GI malignancies $(70.7 \%)$, and the male/female ratio of patients in the group with normal endoscopic findings was approximately $1: 1$. The prevalence of $H$. pylori infection, after excluding the cases that were not tested, was low in the patients with upper GI malignancies $(17.1 \%)$ and was equal to that found in the group with normal endoscopic findings (17.1\%). In contrast, patients with gastroduodenitis or peptic ulcer disease had a higher rate of $H$. pylori infection (43.4\%).

The analysis of the patients' alarm features, as shown in Table 2, revealed a very high specificity (GI bleeding, 96\%; body weight loss, 97.6\%; dysphagia, 99.8\%; at least one alarm feature, 93.8\%), but the sensitivity was relatively low (GI bleeding, 24.4\%; body weight loss, 29.3\%; at least one alarm feature, 60.1\%), especially for dysphagia (14.6\%). Positive predictive values were also low, ranging from $11.6 \%$ to $20.9 \%$, except dysphagia (66.7\%), and the negative predictive value of each alarm feature was very high, ranging from $98.2 \%$ to $99.1 \%$.

Among the 82 patients with upper GI malignancies in our study, as shown in Table 3, AAA 14 (17.1\%), 12 (14.6\%), 52 


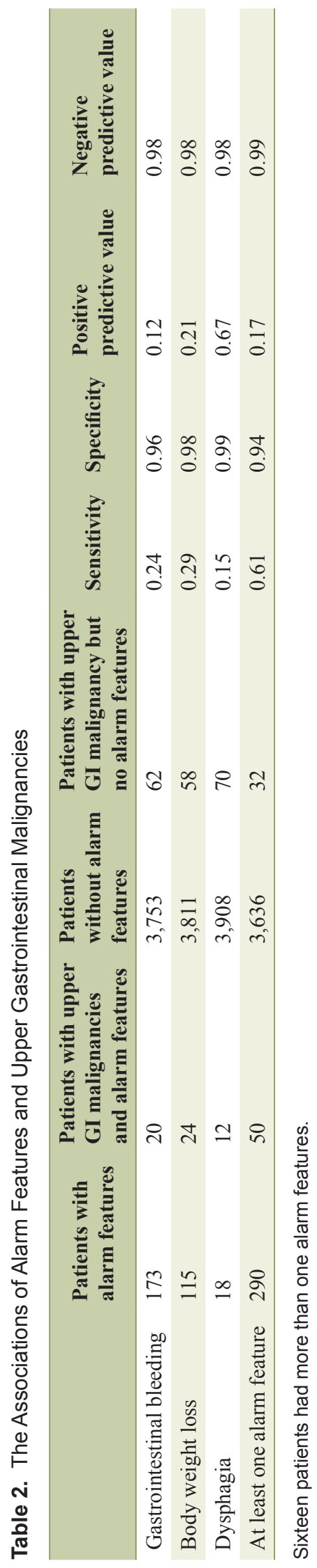

(63.4\%) and four (4.9\%) cases had esophageal cancers, upper (corpus, fundus or upper corpus) gastric cancers, lower (antrum or angularis) gastric cancers, and duodenal cancers, respectively. All the esophageal cancers and gastric cancers were primary squamous cell carcinoma and adenocarcinoma, respectively and the duodenal cancers belonged to ampulla Vater cancers. The patients with esophageal cancers and upper gastric cancers had the highest prevalence rate of at least one alarm feature $(85.7 \%$ and $83.3 \%$ ), and the highest rates were for body weight loss $(50 \%)$ and dysphagia $(50 \%)$. However, the most prevalent alarm feature in patients with lower gastric cancers was GI bleeding (36.5\%). All H. pylori infection in the patients with malignancies happened to those with lower gastric cancers, and it was counted $23.1 \%$ of this group.

An analysis of the relationships between alarm features and stages of gastric cancers is shown in Table 4 . The results showed a significant positive correlation between alarm features and advanced stages of gastric cancers, including the histological and clinical AJCC classification, but not for the sign of GI bleeding. In addition, no correlation was found between alarm features and endoscopic Bormann's classification.

\section{Discussion}

Endoscopy is the most accurate method for diagnosis of important organic GI diseases associated with dyspepsia, including peptic ulcer disease, esophagitis, gastroduodenitis, and especially, GI malignancies. It is vitally important not to delay diagnosis in patients with underlying GI malignancies. However, endoscopy involves some discomfort, significant social inconvenience, and cost. Therefore, it is necessary to identify patients who have a high risk of having cancer so that they can receive immediate endoscopy, which would thus avoid subjecting large numbers of people to an unnecessary endoscopy in daily clinical practice [3].

Recent guidelines suggest that alarm features at any age warrant prompt endoscopy, though an age cut-off of between 50 and 55 years for endoscopy as an initial management strategy has also been widely recommended $[8,9]$. However, a study conducted in Taiwan [10], an area of high background prevalence of gastric cancers, demonstrated that $0.4-5 \%$ of gastric cancers would have been missed if endoscopy had not been offered.

In our study, the mean age and the sex distribution of patients with upper GI malignancies showed a predominance of older patients (64.6 years) and male gender (male/female ratio, 2.4:1). These data imply that elderly males have the highest risk of upper GI malignancies, and endoscopy should be considered regardless of presence of alarm features. The prevalence of upper GI malignancies in our study $(2.1 \%)$ was higher than that of a previous study conducted in Taiwan (1.25\%) [10] and that reported in a Hong Kong study (0.9\%) [11], and the reason for this phenomenon may be related to the more restrictive exclusion criteria in our study, which included reflux esophagitis and previous gastric surgery, and the characteristic of our hospital, a tertiary-care center.

In our study, the percentage of patients with upper GI ma- 
Table 3. The Basic Characteristics of the Individuals With Upper Gastrointestinal Malignancies

\begin{tabular}{|c|c|c|c|c|c|c|}
\hline & \multicolumn{6}{|c|}{ Upper GI malignancies } \\
\hline & $\begin{array}{l}\text { Esophageal cancers } \\
(\mathrm{n}=14,17.1 \%)\end{array}$ & $\begin{array}{l}\text { Upper gastric } \\
\text { cancers } \\
(n=12,14.6 \%)\end{array}$ & $\begin{array}{l}\text { Lower gastric } \\
\text { cancers } \\
(\mathrm{n}=52,63.4 \%)\end{array}$ & $\begin{array}{l}\text { Duodenal } \\
\text { cancers } \\
(n=4,4.9 \%)\end{array}$ & $\begin{array}{l}\text { Total } \\
(n=82)\end{array}$ & P-value \\
\hline Age (years) & $63.14 \pm 13.92$ & $67.83 \pm 13.89$ & $63.19 \pm 13.37$ & $77.50 \pm 5.20$ & & $0.122^{\mathrm{a}}$ \\
\hline \multicolumn{7}{|l|}{ Gender } \\
\hline Male & $12(85.7 \%)$ & $10(17.2 \%)$ & $32(61.5 \%)$ & $4(100 \%)$ & $58(70.7 \%)$ & $0.102^{\mathrm{b}}$ \\
\hline \multicolumn{7}{|l|}{ H. pylori infection } \\
\hline Positive & 0 & $0^{\#}$ & $12(23.1 \%)^{\#}$ & 0 & $12(17.1 \%)$ & $0.171^{\mathrm{b}}$ \\
\hline Excluded numbers & 12 & 0 & 0 & 0 & & \\
\hline \multicolumn{7}{|l|}{ Alarm features } \\
\hline GI bleeding & $1(7.1 \%)$ & 0 & $19(36.5 \%)$ & 0 & 18 & $0.008^{b}$ \\
\hline
\end{tabular}

aKruskal-Wallis one-way ANOVA test. bPearson's Chi-square test. Upper gastric cancers is defined as cancers in the cardia, fundus or upper corpus; lower gastric cancers is defined as cancers in antrum or angularis. ${ }^{\mathrm{P}}=0.062$ analyzed by Fisher's exact test. Six patients had more than one alarm features.

lignancies and at least one alarm feature was $60.1 \%$. Among the three alarm features, body weight loss had the highest sensitivity, though it was only $29.3 \%$, and dysphagia had the lowest sensitivity (14.6\%). In contrast, the specificity and negative predictive value in our study were very high, ranging from $93.8 \%$ to $99.8 \%$, for individual alarm features as well as for at least one alarm feature. The positive predictive value of dysphagia $(66.7 \%)$ was somewhat better than those of the other alarm features, especially in patients with esophageal cancers. These results indicate that only a small proportion of the patients with alarm symptoms were found to have cancers. Thus, the sensitivity and positive predictive value of the alarm symptoms were disappointingly low, and this finding was consistent with the results of previous studies $[2-4,12,13]$. The very high specificity and negative predictive value might reflect the low prevalence of upper GI malignancies in our patient population, but there was no specific attribute of any of the alarm features which could be used to exclude potential malignancies.

Regarding the types of upper GI malignancies and their relationships with alarm features, the malignancies of esophagus and upper stomach were likely to be associated with the alarm features of body weight loss and dysphagia, which might be explained by the close proximity of these two types of malignancies. The lower gastric cancers, defined as cancers in antrum or angularis, were more likely to be associated with GI bleeding, and interestingly, showed a higher percentage of $H$. pylori infection than those of upper gastric cancers, although the difference was non-significant.

Several studies showed that in patients with upper GI malignancies, the occurrence of alarm symptoms usually indicated more advanced cancers with worse prognosis $[14,15]$. Moreover, in patients with gastric cancers, body weight loss and melena are certainly indices of more advanced cancer, so it is essential to develop methods which can identify gastric cancers at an early stage [16]. Unfortunately, the relationship between stages of cancers and the presence of alarm symptoms has not yet been fully elucidated.

In the present study, we analyzed the relationship between each stage of various gastric cancers and alarm features. The signs of body weight loss and dysphagia were found to be significantly correlated with advanced stages, including the histological and AJCC classification, of gastric cancers. The only exception was the sign of GI bleeding, which tends to prompt diagnosis of early gastric cancer and therefore may have a better prognosis. The endoscopic pictures of gastric cancers, known as the Bormann's classification, did not appear to be related to alarm features in our study.

There were some limitations in our study. Firstly, varying thresholds for the determination of alarm features were applied. Secondly, this was a hospital-based study and only included patients who were referred for endoscopy. The exclusion of elderly patients who had alarm features, but were unable to receive endoscopy due to personal or physical reasons, may have decreased both the sensitivity and positive predictive value of alarm features. However, young patients without alarm features may have been less likely to undergo endoscopy, and this may have decreased both the specificity and negative predictive value a little. Further researches in representative samples of the general population are needed to confirm these results.

\section{Conclusion}

In the present study, alarm features had a disappointingly low diagnostic value for upper GI malignancies. However, the sign 


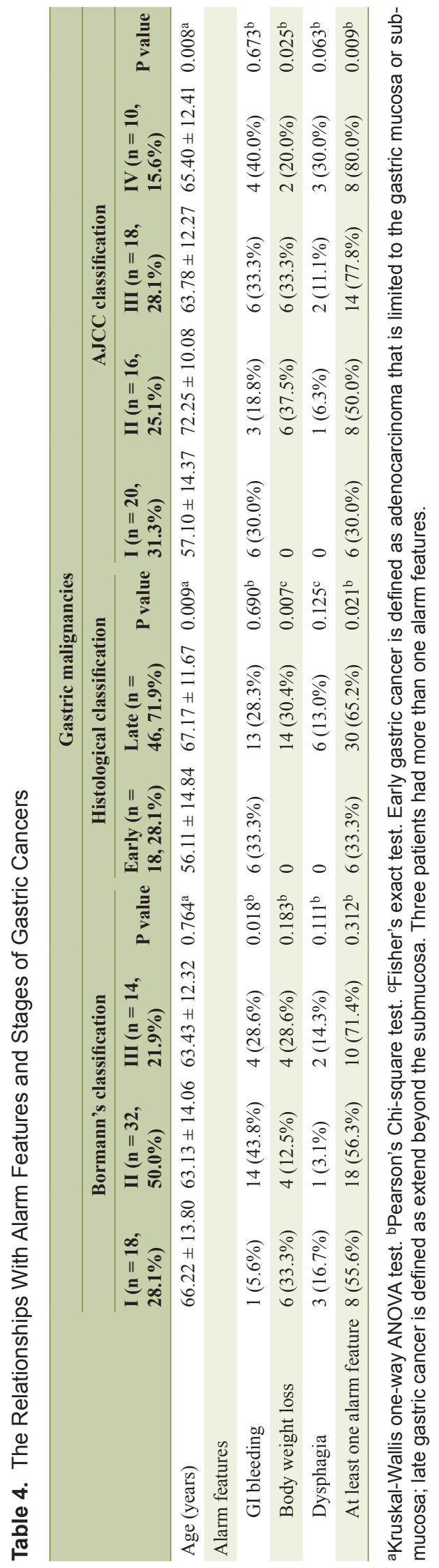

of dysphagia for esophageal or upper gastric cancer, and the sign of GI bleeding for the detection of early gastric cancer, can still be applied in clinical practice in populations that have a high risk of malignancies. The presences of alarm features were correlated with the more advanced histological and clinical stages of gastric malignancies, but not for the endoscopic appearance.

\section{References}

1. Talley NJ, Vakil NB, Moayyedi P. American gastroenterological association technical review on the evaluation of dyspepsia. Gastroenterology. 2005;129(5):1756-1780.

2. Vakil N, Moayyedi P, Fennerty MB, Talley NJ. Limited value of alarm features in the diagnosis of upper gastrointestinal malignancy: systematic review and meta-analysis. Gastroenterology. 2006;131(2):390-401; quiz 659360.

3. Fransen GA, Janssen MJ, Muris JW, Laheij RJ, Jansen JB. Meta-analysis: the diagnostic value of alarm symptoms for upper gastrointestinal malignancy. Aliment Pharmacol Ther. 2004;20(10):1045-1052.

4. Wallace MB, Durkalski VL, Vaughan J, Palesch YY, Libby ED, Jowell PS, Nickl NJ, et al. Age and alarm symptoms do not predict endoscopic findings among patients with dyspepsia: a multicentre database study. Gut. 2001;49(1):29-34.

5. Alexander HR, Kelsen PD, Tepper JB. Cancer of the Stomach. Chapter 1021. In: De Vita, editor. Cancer: Principles and Practices Oncology. 5th ed. Philadelphia: Lippincott; 1997.

6. Murakami T. Pathomorphological diagnosis. Definition and gross classification of early gastric cancer. Gann Monogr Cancer Res. 1971;11:53.

7. Edge SB, Byrd DR, Compton CC, et al (Eds). American Joint Committee on Cancer Staging Manual, 7th. New York: Springer; 2010. p.117.

8. National Institute for Health and Clinical Excellence. Dyspepsia: managing dyspepsia in adults in primary care, 2004. www.nice.org.uk/Guidance/CG17.

9. Talley NJ, Vakil N. Guidelines for the management of dyspepsia. Am J Gastroenterol. 2005;100(10):2324-2337.

10. Liou JM, Lin JT, Wang HP, Huang SP, Lee YC, Shun CT, Lin MT, et al. The optimal age threshold for screening upper endoscopy for uninvestigated dyspepsia in Taiwan, an area with a higher prevalence of gastric cancer in young adults. Gastrointest Endosc. 2005;61(7):819-825.

11. Sung JJ, Lao WC, Lai MS, Li TH, Chan FK, Wu JC, Leung VK, et al. Incidence of gastroesophageal malignancy in patients with dyspepsia in Hong Kong: implications for screening strategies. Gastrointest Endosc. 2001;54(4):454-458.

12. Vakil NB, Traxler B, Levine D. Dysphagia in patients with erosive esophagitis: prevalence, severity, and response to proton pump inhibitor treatment. Clin Gastroenterol Hepatol. 2004;2(8):665-668.

13. Bai Y, Li ZS, Zou DW, Wu RP, Yao YZ, Jin ZD, Ye P, et al. Alarm features and age for predicting upper gastro- 
intestinal malignancy in Chinese patients with dyspepsia with high background prevalence of Helicobacter pylori infection and upper gastrointestinal malignancy: an endoscopic database review of 102,665 patients from 1996 to 2006. Gut. 2010;59(6):722-728.

14. Siersema PD, Dees J, Tilanus HW, Kok TC, Hordijk ML, van Blankenstein M. Early detection and treatment of oesophageal and gastric cancer. The Rotterdam Oesophageal Tumour Study Group. Neth J Med. 1995;47(2):76-86.
15. Kubota H, Kotoh T, Masunaga R, Dhar DK, Shibakita M, Tachibana M, Kohno H, et al. Impact of screening survey of gastric cancer on clinicopathological features and survival: retrospective study at a single institution. Surgery. 2000;128(1):41-47.

16. Boldys H, Marek TA, Wanczura P, Matusik P, Nowak A. Even young patients with no alarm symptoms should undergo endoscopy for earlier diagnosis of gastric cancer. Endoscopy. 2003;35(1):61-67. 